\title{
Measurement and Prediction Method of Compressibility Factor at High Temperature and High Pressure
}

\author{
Xiaoxun Zhu, Bochao Xu, and Zhonghe Han \\ Department of Power Engineering, North China Electric Power University, Baoding 071003, China \\ Correspondence should be addressed to Xiaoxun Zhu; zhuxiaoxun@hotmail.com
}

Received 3 March 2016; Accepted 14 April 2016

Academic Editor: Giuseppe Vairo

Copyright (C) 2016 Xiaoxun Zhu et al. This is an open access article distributed under the Creative Commons Attribution License, which permits unrestricted use, distribution, and reproduction in any medium, provided the original work is properly cited.

\begin{abstract}
In order to get the compressibility factor $Z$ of working fluid under different conditions, experimental measurement method of $Z$ under high pressure and high temperature and data mining method were studied in this paper. Experimental measurement method based on real gas state equation and prediction method based on Least Squares Support Vector Machine were proposed. First, an experimental method for measuring $Z$ at high temperature and high pressure was designed; in this method the temperature, pressure, and density (mass and volume) of corresponding state were measured and substituted into the actual gas equation of state, and then $Z$ can be calculated. Meanwhile, in order to obtain continuous value in $T$ - $p$ plane, Squares Support Vector Machines are introduced to establish the prediction model of $Z$. Take Hexamethyldisiloxane, for example; the experimental data of $Z$ was obtained using the experimental method. Meanwhile the prediction model of $Z$, which can be used as calculation function of $Z$, was established based on those experimental data, and the $Z$ ( $T: 500 \mathrm{~K} \sim 800 \mathrm{~K}, p: 1.3 \mathrm{MPa} 2.25 \mathrm{MPa}$ ) was calculated by using this calculation function. By comparison with this published data, it was found that the average relative error was $2.14 \%$.
\end{abstract}

\section{Introduction}

Compressibility factor ( $Z$-factor) [1-3] is an important parameter of thermal physical properties and is often used to calculate other physical parameters. $Z$-factor changes with temperature and pressure. In engineering calculation, the $Z$ factor values under different $(T, p)$ are needed. However, for the new refrigerant or different batches, these data are not complete, especially at high temperature and high pressure.

Currently the method based on the law of corresponding states is a common mean $[4,5]$. However, this approach is an empirical method. At high temperature and high pressure region, sometimes the error of this method will be serious. Therefore, it is very necessary to devise a method to measure and calibrate $Z$-factor in the region of high temperature and high pressure.

It is direct to calculate $Z$-factor by actual gas state equation [6]. It can be known from the equation that $Z$-factor and density (mass $m$ and volume $V$ ) are corresponding when temperature $T$ and pressure $p$ were given. Then, $Z$-factor can be solved by $T, p, m$, and $V$. So it is the premise to obtain $m$ and $V$ value under the condition of $(T, p)$.
Based on the above analysis, a measurement method of $Z$-factor is designed based on actual gas state equation. In this experiment, a given quality of working fluid is heated in a container, whose volume is constant, and the changes of $T$ and $p$ are recorded, and $m$ and $V$ at different $(T, p)$ are got; finally $Z$-factor can be obtained correspondingly. This method is simple, and the precision can be guaranteed at the high temperature and high pressure region, even at supercritical region.

However, $Z$-factor obtained by experiment is discrete in particular $(T, p)$ status, and the needs of engineering applications cannot be met. In general, empirical formula established by the experimental data is used to meet the practical engineering application. And the accuracy of the empirical formula is the key. In this paper, $Z$-factor prediction method based on Least Squares Support Vector Machine is proposed. In this method, LSSVM is used to replace the traditional empirical formula to establish prediction function in $T$ - $p$ plane.

In this paper, Hexamethyldisiloxane (MM) was taken, for example, by using this method; $Z$-factor at different $(T, p)$ was measured, and $Z$-factor prediction function of $\mathrm{MM}$ 
was established. $Z$-factor values of MM (T: $500 \mathrm{~K} \sim 800 \mathrm{~K}, p$ : $1.3 \mathrm{MPa} 2.25 \mathrm{MPa}$ ) and the $Z$ - $p$ graph were obtained by the prediction function. By comparison with the published data, it was found that the average relative error was $2.14 \%$. The effectiveness of this method and precision of the function were verified.

\section{Design Density Measurement Method}

2.1. The Scheme of Experiment. By the real gas state equation [7],

$$
p V=Z n R T
$$

It can be got that

$$
Z=\frac{M V p}{m R T}=\frac{M p}{\rho R T} .
$$

It can be seen from formula (2), as long as the corresponding temperature, pressure, and density (mass and volume) can be measured, that the corresponding $Z$-factor can be calculated.

In the region of high temperature and high pressure, especially in a supercritical state, because of sealing, the change of mass and volume tends to bring measurement error. So in this experiment, the mass and volume are fixed. In this method a certain mass working fluid is added to a container, whose volume is constant. The working fluid in the container will be at superheated or supercritical state if the container is heated continuously. During the heating process, the changes of $T$ and $p$ should be recorded correspondingly.

Further analysis showed that, beside the measuring working fluid, there is also air in the container. So $p$ is the total pressure of measuring working fluid and air, but not the actual pressure of this measured medium. For the ideal gas, the pressure of component can be solved by Dalton law of partial pressure $[8,9]$ :

$$
\begin{aligned}
& \frac{p_{i}}{p}=\frac{n_{i}}{n}=x_{i}, \\
& p_{i}=x_{i} p,
\end{aligned}
$$

where $p$ is total pressure, $p_{i}$ is partial pressure of the $i$ th component, $n$ is total molar mass, $n_{i}$ is molar mass of the $i$ th component, and $x_{i}$ is the molar mass fraction of the $i$ th component.

However, this law considers that $Z$-factor is constant equal to 1 . For the actual gas, $Z$-factor is changed with temperature and pressure. So the $Z$-factor of each component in each state must be got before calculating the partial pressure of actual gas. Obviously, this contradicts with the purpose of this paper.

Therefore, in order to eliminate the influence of other components (air) on the pressure measurement of working fluid, the container must be vacuumed before being heated. Then $p$ is the actual pressure of working fluid. Furthermore the corresponding density $\rho$ of $(T, p)$ points can be got.

The corresponding $Z$-factor can be obtained by substituting $T$, $p$, and $\rho$ to formula (2).

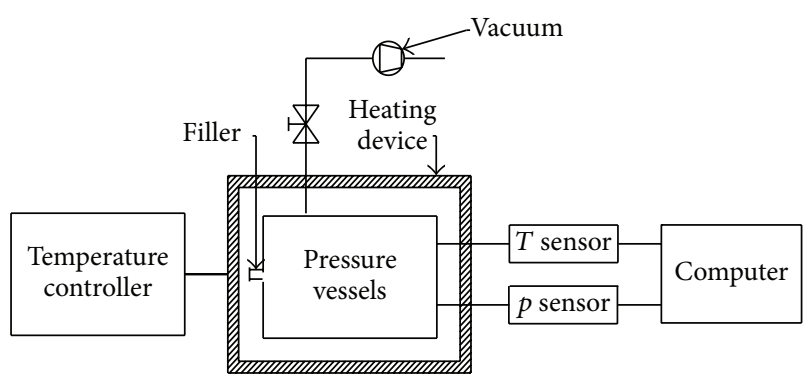

Figure 1: Experimental apparatus.

2.2. Experimental Devices. The whole experiment requires the following devices: the measured medium, pressure vessels, temperature sensors, pressure sensors, electronic scale, heating device, temperature control device, vacuum pump, and so on. The experimental structure was shown in Figure 1.

As shown in Figure 1, the temperature control device heats the container through adjusting the temperature of heating equipment. The temperature and pressure of the container are measured by the temperature and pressure sensors.

\section{Experiment}

3.1. Experimental Material. As an important organic working medium, MM is increasingly applied to ORC system with its excellent thermodynamic properties under the state of high temperature and high pressure [10, 11]. Take MM, for example; this paper used the experimental method to measure its density in the state of high temperature and high pressure ( $T$ : $500 \mathrm{~K} \sim 850 \mathrm{~K}, p: 1.2 \mathrm{MPa} 2.1 \mathrm{MPa})$ and calculated the corresponding $Z$-factor.

$\mathrm{K}$ type thermocouples and pressure gauge were used to measure the temperature and pressure, the use of electronic scales measuring refrigerant mass. At the same time, it used resistance box as the heating device and used temperature controller to adjust the heating temperature. Pressure vessel, whose volume is constant of $1084 \mathrm{~mL}$, was made of stainless steel.

3.2. Error Analysis. First of all, the experimental uncertainty should be analyzed.

The accuracy of $\mathrm{K}$ type thermocouple is $0.75 \%$, the accuracy of pressure gauge is $0.4 \%$, and the electronic precision is $30 \mathrm{~kg} \pm 0.1 \mathrm{~g}$, so the uncertainty of this experimental system is estimated to be $0.87 \%$.

At the same time, the relative error and the mean relative error were calculated by the following formula:

$$
\begin{aligned}
\delta_{i} & =\frac{\left|Z_{i}-Z_{i s}\right|}{Z_{i s}} \times 100, \\
\delta & =\frac{1}{n} \sum_{i=1}^{n} \frac{\left|Z_{i}-Z_{i s}\right|}{Z_{i s}} \times 100,
\end{aligned}
$$

where $Z_{i}$ is measurement (computing) value and $Z_{i s}$ is published values. 
TABLE 1: $Z$-factor obtained by experiment $\left(\rho=48.43 \mathrm{~kg} / \mathrm{m}^{3}\right)$.

\begin{tabular}{lcccc}
\hline$p / \mathrm{MPa}$ & $T / \mathrm{K}$ & $Z_{i}$ & $Z_{\text {is }}$ & $\delta / \%$ \\
\hline 1.24 & 580.17 & 0.861 & 0.863 & 0.27 \\
1.3 & 599.39 & 0.874 & 0.879 & 0.57 \\
1.34 & 613.1 & 0.880 & 0.889 & 0.93 \\
1.4 & 632.25 & 0.892 & 0.901 & 0.99 \\
1.44 & 646.79 & 0.897 & 0.910 & 1.41 \\
1.5 & 667.11 & 0.906 & 0.920 & 1.59 \\
1.54 & 680.08 & 0.912 & 0.927 & 1.55 \\
1.6 & 700.41 & 0.920 & 0.936 & 1.64 \\
1.66 & 721.23 & 0.927 & 0.944 & 1.78 \\
1.7 & 733.03 & 0.934 & 0.948 & 1.46 \\
1.74 & 746.88 & 0.938 & 0.953 & 1.52 \\
1.8 & 765.7 & 0.947 & 0.959 & 1.25 \\
1.84 & 776.72 & 0.954 & 0.962 & 0.82 \\
1.88 & 787.33 & 0.962 & 0.965 & 0.33 \\
1.9 & 793.8 & 0.964 & 0.967 & 0.28 \\
1.94 & 804.93 & 0.971 & 0.970 & 0.11 \\
1.96 & 811.53 & 0.973 & 0.972 & 0.14 \\
1.98 & 817.23 & 0.976 & 0.973 & 0.31 \\
2 & 822.28 & 0.980 & 0.974 & 0.57 \\
2.02 & 829.58 & 0.981 & 0.976 & 0.50 \\
2.04 & 831.4 & 0.988 & 0.976 & 1.24 \\
2.06 & 835.31 & 0.993 & 0.977 & 1.66 \\
2.08 & 838.53 & 0.999 & 0.978 & 2.19 \\
2.1 & 842.3 & 1.004 & 0.979 & 2.62 \\
\hline & & & &
\end{tabular}

3.3. Analysis of the Experimental Process and Results. First of all, we added $52.5 \mathrm{~g}, 54.4 \mathrm{~g}, 58.4 \mathrm{~g}, 64.8 \mathrm{~g}$, and $71.2 \mathrm{~g} \mathrm{MM}$ to each pressure vessel, and the corresponding densities were $48.43 \mathrm{~kg} / \mathrm{m}^{3}, 50.18 \mathrm{~kg} / \mathrm{m}^{3}, 53.87 \mathrm{~kg} / \mathrm{m}^{3}, 59.78 \mathrm{~kg} / \mathrm{m}^{3}$, and $65.68 \mathrm{~kg} / \mathrm{m}^{3}$.

Heat and record $T$ and $p$ after overheating state. Measurement and calculation results of $Z$-factor are shown in Tables 1-5. This paper uses the PEFPROP property search software to query $Z$ of $M M$, and the error analyses are shown in Tables $1-5$.

It can be seen from Tables 1-5, in this experiment, that the relative error is small, and the average relative error is about $2.25 \%$. For the supercritical state is difficult to measure, the result is also very good.

Beside the fact that the measurement of $T$ and $p$ may lead to errors, on the other hand, the tightness of experiment device, which results in a small amount of leakage in the heating process, will lead to the changes of density and cause the error of $Z$-factor. But the effect is small, which can be enhanced by the sealing means.

\section{The Prediction Model of $Z$-Factor Based on LSSVM}

$Z$-factor of refrigerants can be obtained accurately by this experimental method. And the precision is high even at high temperature and high pressure conditions. However, $Z$-factor
TABLE 2: $Z$-factor obtained by experiment $\left(\rho=50.18 \mathrm{~kg} / \mathrm{m}^{3}\right)$.

\begin{tabular}{|c|c|c|c|c|}
\hline$p / \mathrm{MPa}$ & $T / \mathrm{K}$ & $Z_{i}$ & $Z_{i s}$ & $\delta / \%$ \\
\hline 1.1 & 526.34 & 0.812 & 0.800 & 1.62 \\
\hline 1.16 & 544.42 & 0.828 & 0.822 & 0.72 \\
\hline 1.2 & 558.92 & 0.835 & 0.840 & 0.58 \\
\hline 1.24 & 570.24 & 0.845 & 0.850 & 0.57 \\
\hline 1.3 & 589.27 & 0.858 & 0.867 & 1.10 \\
\hline 1.36 & 607.55 & 0.870 & 0.881 & 1.25 \\
\hline 1.4 & 620.78 & 0.877 & 0.89 & 1.57 \\
\hline 1.44 & 634.35 & 0.882 & 0.900 & 1.91 \\
\hline 1.5 & 655.67 & 0.889 & 0.912 & 2.53 \\
\hline 1.54 & 667.98 & 0.896 & 0.919 & 2.45 \\
\hline 1.6 & 682.84 & 0.911 & 0.925 & 1.57 \\
\hline 1.66 & 709.82 & 0.909 & 0.938 & 3.10 \\
\hline 1.7 & 724.66 & 0.912 & 0.944 & 3.41 \\
\hline 1.74 & 735.99 & 0.919 & 0.948 & 3.08 \\
\hline 1.8 & 752.87 & 0.929 & 0.954 & 2.56 \\
\hline 1.84 & 763.83 & 0.936 & 0.957 & 2.18 \\
\hline 1.88 & 775.17 & 0.943 & 0.961 & 1.87 \\
\hline 1.9 & 779.83 & 0.947 & 0.962 & 1.55 \\
\hline 1.92 & 785.47 & 0.950 & 0.964 & 1.40 \\
\hline 1.94 & 790.73 & 0.954 & 0.965 & 1.18 \\
\hline 1.96 & 796.34 & 0.957 & 0.967 & 1.03 \\
\hline 1.98 & 803.95 & 0.957 & 0.969 & 1.19 \\
\hline 2 & 808.22 & 0.962 & 0.970 & 0.83 \\
\hline 2.02 & 814.7 & 0.964 & 0.972 & 0.81 \\
\hline 2.04 & 818.83 & 0.969 & 0.973 & 0.43 \\
\hline 2.06 & 823.71 & 0.972 & 0.974 & 0.18 \\
\hline 2.08 & 827.47 & 0.977 & 0.975 & 0.25 \\
\hline 2.1 & 830.1 & 0.983 & 0.975 & 0.83 \\
\hline
\end{tabular}

obtained by the experiment method above is discrete. In practice, all $Z$-factors on $T$ - $p$ plane are needed. Moreover, all the experimental data are on the isodense line. That is difficult for the practical application.

Obviously, it is extremely difficult, even impossible, to get all $Z$-factors on $T$ - $p$ plane by using the experiment only. So this paper proposes a prediction method of $Z$-factor based on LSSVM ( $Z$-LSSVM). And a calculation function of $Z$-factor on the plane is established; meanwhile $Z$-factor of $\mathrm{MM}$ on $T-p$ plane had been got by using this function.

4.1. Least Squares Support Vector Machine. SVR [12, 13] theory was first proposed by Vapnik in the 1990s. SVR is a machine learning technique based on the structural risk minimization principle, which solves the problem of local minima and nonlinear problem. On the base of SVR, the Least Square Support Vector Machine was proposed by Suykens $[14,15]$ to improve the solution speed and convergence precision.

For a given training set,

$T=\left\{\left(x_{i}, y_{i}\right) \mid i=1,2,3, \ldots, n\right\}, \quad x_{i} \in R^{n}, y_{i} \in R$.

Solving for $x$ and $y$ mapping function $f(x)$,

$$
y=f(x)=\omega^{T} \varphi(x)+b .
$$


TABLE 3: $Z$-factor obtained by experiment $\left(\rho=53.87 \mathrm{~kg} / \mathrm{m}^{3}\right)$.

\begin{tabular}{lcccc}
\hline$p / \mathrm{MPa}$ & $T / \mathrm{K}$ & $Z_{i}$ & $Z_{i s}$ & $\delta / \%$ \\
\hline 1.14 & 527 & 0.783 & 0.792 & 1.059 \\
1.2 & 539.8 & 0.805 & 0.806 & 0.118 \\
1.24 & 549.34 & 0.817 & 0.817 & 0.084 \\
1.3 & 566.14 & 0.832 & 0.836 & 0.478 \\
1.34 & 577.28 & 0.841 & 0.847 & 0.716 \\
1.4 & 595.84 & 0.851 & 0.864 & 1.52 \\
1.44 & 606.92 & 0.859 & 0.873 & 1.56 \\
1.5 & 625.22 & 0.869 & 0.887 & 2.01 \\
1.54 & 638.1 & 0.874 & 0.896 & 2.41 \\
1.6 & 655.82 & 0.883 & 0.906 & 2.53 \\
1.64 & 669.71 & 0.887 & 0.915 & 3.04 \\
1.7 & 687.27 & 0.896 & 0.923 & 3.01 \\
1.74 & 700.53 & 0.899 & 0.930 & 3.28 \\
1.8 & 722.99 & 0.902 & 0.940 & 4.10 \\
1.84 & 736.18 & 0.905 & 0.945 & 4.27 \\
1.88 & 750.19 & 0.907 & 0.951 & 4.55 \\
1.9 & 757.75 & 0.908 & 0.954 & 4.78 \\
1.92 & 764.96 & 0.909 & 0.956 & 4.93 \\
1.94 & 770.57 & 0.912 & 0.958 & 4.82 \\
1.96 & 776.84 & 0.914 & 0.960 & 4.81 \\
1.98 & 783.78 & 0.915 & 0.962 & 4.91 \\
2 & 790.64 & 0.916 & 0.964 & 4.99 \\
2.02 & 798.29 & 0.916 & 0.967 & 5.19 \\
2.04 & 804 & 0.919 & 0.968 & 5.09 \\
2.06 & 809.14 & 0.922 & 0.970 & 4.91 \\
2.08 & 814.84 & 0.924 & 0.971 & 4.81 \\
2.1 & 820.83 & 0.926 & 0.973 & 4.75 \\
\hline & & & & \\
& 54 & &
\end{tabular}

In the formula, $\omega$ is the weight vector, $b$ is the offset, and $\varphi(x)$ is a mapping function. The equation can be used to estimate the unknown nonlinear functions.

Based on the principle of structural risk minimization, constructing, and solving the optimization problem:

$$
\begin{array}{cl}
\text { Min } & J(\omega, e, b)=\frac{1}{2} \omega^{T} \omega+\frac{1}{2} \gamma \sum_{i=1}^{n} e_{i}^{2} \\
\text { s.t. } & y_{i}=\omega^{T} \varphi\left(x_{i}\right)+b+e_{i}, \quad i=1,2, \ldots, n \\
& e_{i} \geq 0, \quad i=1,2, \ldots, l .
\end{array}
$$

In the formula, $\gamma$ is the penalty coefficient, which mainly controls the punishment degree, and $J(\omega, e, b)$ is the loss function.

In order to solve $\omega$ and $e$, the Lagrange method is introduced. The definition of Lagrange function is as follows:

$$
\begin{aligned}
L(\omega, b, e, \alpha, \gamma)= & \frac{1}{2} \omega^{T} \omega+\frac{1}{2} \gamma \sum_{i=1}^{n} e_{i}^{2} \\
& -\sum_{i=1}^{n} \alpha_{i}\left\{\left(\omega^{T} \varphi\left(x_{i}\right)\right)+b+e_{i}-y_{i}\right\} .
\end{aligned}
$$

In the formula, $\alpha_{i}$ is the Lagrange multiplier.
TABLE 4: $Z$-factor obtained by experiment $\left(\rho=59.78 \mathrm{~kg} / \mathrm{m}^{3}\right)$.

\begin{tabular}{lcccc}
\hline$p / \mathrm{MPa}$ & $T / \mathrm{K}$ & $Z_{i}$ & $Z_{i s}$ & $\delta / \%$ \\
\hline 1.06 & 490.63 & 0.705 & 0.707444 & 0.33 \\
1.1 & 499.84 & 0.718 & 0.726835 & 1.19 \\
1.14 & 507.77 & 0.733 & 0.739998 & 0.98 \\
1.2 & 521.9 & 0.750 & 0.764312 & 1.82 \\
1.24 & 530.83 & 0.762 & 0.77743 & 1.94 \\
1.3 & 544.41 & 0.779 & 0.795667 & 2.06 \\
1.34 & 553.94 & 0.789 & 0.807694 & 2.26 \\
1.4 & 568.41 & 0.804 & 0.824353 & 2.49 \\
1.44 & 578.43 & 0.812 & 0.83508 & 2.71 \\
1.5 & 594.19 & 0.824 & 0.850752 & 3.16 \\
1.54 & 603.74 & 0.832 & 0.859029 & 3.09 \\
1.6 & 616.63 & 0.847 & 0.868892 & 2.54 \\
1.64 & 630.24 & 0.849 & 0.880 & 3.54 \\
1.7 & 647.99 & 0.856 & 0.893 & 4.16 \\
1.74 & 659.28 & 0.861 & 0.901 & 4.38 \\
1.8 & 675.64 & 0.869 & 0.911 & 4.51 \\
1.84 & 687.28 & 0.874 & 0.917 & 4.73 \\
1.88 & 698.82 & 0.878 & 0.923 & 4.90 \\
1.9 & 702.64 & 0.883 & 0.925 & 4.58 \\
1.92 & 706.76 & 0.887 & 0.927 & 4.33 \\
1.94 & 710.87 & 0.891 & 0.929 & 4.08 \\
1.96 & 717.54 & 0.891 & 0.932 & 4.33 \\
1.98 & 722.42 & 0.894 & 0.934 & 4.23 \\
2 & 726.69 & 0.898 & 0.936 & 4.01 \\
2.02 & 735.27 & 0.897 & 0.940 & 4.60 \\
2.04 & 733.03 & 0.908 & 0.938 & 3.18 \\
2.06 & 740.94 & 0.907 & 0.942 & 3.64 \\
2.08 & 743.88 & 0.913 & 0.943 & 3.19 \\
2.1 & 751.33 & 0.912 & 0.946 & 3.56 \\
\hline & & & &
\end{tabular}

Optimizing the formula according to Karush-KuhnTucker (KKT),

$$
\begin{aligned}
\frac{\partial L}{\partial \omega} & =0 \longrightarrow \\
\omega & =\sum_{i=1}^{n} \alpha_{i} \varphi\left(x_{i}\right), \\
\frac{\partial L}{\partial b} & =0 \longrightarrow \\
\sum_{i=1}^{n} \alpha_{i} & =0, \\
\frac{\partial L}{\partial e_{i}} & =0 \longrightarrow \\
\gamma \delta_{i} e_{i} & =\alpha_{i}, \\
\frac{\partial L}{\partial \alpha_{i}} & =0 \longrightarrow \\
\left(\omega^{T} \varphi\left(x_{i}\right)\right)+b+e_{i}-y_{i} & =0, \\
{\left[\begin{array}{c}
0 \\
1_{v}^{T} \\
\left.\Omega+\frac{I}{\lambda}\right]
\end{array}\right]\left[\begin{array}{l}
b \\
\alpha
\end{array}\right] } & =\left[\begin{array}{l}
0 \\
y
\end{array}\right] .
\end{aligned}
$$


TABLE 5: $Z$-factor obtained by experiment $\left(\rho=65.68 \mathrm{~kg} / \mathrm{m}^{3}\right)$.

\begin{tabular}{|c|c|c|c|c|}
\hline$p / \mathrm{MPa}$ & $T / \mathrm{K}$ & $Z_{i}$ & $Z_{i s}$ & $\delta / \%$ \\
\hline 1.06 & 481.13 & 0.654 & 0.662 & 1.16 \\
\hline 1.1 & 487.72 & 0.670 & 0.676 & 0.87 \\
\hline 1.14 & 494.86 & 0.684 & 0.691 & 0.99 \\
\hline 1.2 & 505.62 & 0.705 & 0.707 & 0.34 \\
\hline 1.24 & 511.01 & 0.721 & 0.718 & 0.33 \\
\hline 1.3 & 520.25 & 0.742 & 0.732 & 1.43 \\
\hline 1.34 & 528.55 & 0.753 & 0.747 & 0.83 \\
\hline 1.4 & 541.32 & 0.768 & 0.768 & 0.04 \\
\hline 1.44 & 550.09 & 0.778 & 0.781 & 0.46 \\
\hline 1.5 & 562.88 & 0.792 & 0.798 & 0.86 \\
\hline 1.54 & 571.83 & 0.800 & 0.810 & 1.21 \\
\hline 1.6 & 584.89 & 0.813 & 0.825 & 1.47 \\
\hline 1.64 & 592.71 & 0.822 & 0.832 & 1.27 \\
\hline 1.7 & 607.21 & 0.832 & 0.847 & 1.86 \\
\hline 1.74 & 617.13 & 0.837 & 0.857 & 2.26 \\
\hline 1.8 & 630.52 & 0.848 & 0.868 & 2.32 \\
\hline 1.84 & 640.9 & 0.853 & 0.877 & 2.73 \\
\hline 1.88 & 650.66 & 0.858 & 0.884 & 2.93 \\
\hline 1.9 & 656.59 & 0.860 & 0.889 & 3.28 \\
\hline 1.92 & 661.41 & 0.862 & 0.892 & 3.34 \\
\hline 1.94 & 663.59 & 0.868 & 0.893 & 2.74 \\
\hline 1.96 & 668.04 & 0.871 & 0.896 & 2.71 \\
\hline 1.98 & 677.29 & 0.868 & 0.903 & 3.81 \\
\hline 2 & 682.57 & 0.870 & 0.906 & 3.95 \\
\hline 2.02 & 685.44 & 0.875 & 0.907 & 3.54 \\
\hline 2.04 & 688.7 & 0.880 & 0.909 & 3.22 \\
\hline 2.06 & 691.45 & 0.885 & 0.910 & 2.79 \\
\hline 2.08 & 695.35 & 0.888 & 0.912 & 2.62 \\
\hline 2.1 & 699.52 & 0.892 & 0.914 & 2.52 \\
\hline
\end{tabular}

Among them,

$$
\begin{aligned}
y & =\left[y_{1}, \ldots, y_{n}\right], \\
1_{v} & =[1, \ldots, 1], \\
\alpha & =\left[\alpha_{1}, \ldots, \alpha_{n}\right] .
\end{aligned}
$$

The transformation by kernel function can be expressed as

$$
\Omega_{i j}=\varphi\left(x_{i}\right)^{T} \varphi\left(x_{j}\right)=K\left(x_{i}, x_{j}\right) .
$$

The fitting function LSSVM can be expressed as

$$
f(x)=\sum_{i=1}^{n} \alpha_{i} k\left(x, x_{i}\right)+b
$$

4.2. The Prediction Method of Z-Factor Based on LSSVM. Prediction method is very important and must have the following characteristics:

(1) the ability to solve the small-scale sample problem [16]: the data obtained by experiment is limited, so the method should find out the variation from smallscale sample set;

(2) the ability on nonlinear problem [16]: $Z$-factor changes with $T$ and $p$, and its relationship with $T$ and $p$ is nonlinear. So, the prediction model must have the ability to mine the nonlinear relationship.

Through the above analysis, LSSVM is a suitable method for the prediction of $Z$-factor. The prediction of $Z$-factor is to establish the function of $Z$-factor with $T$ and $p$ :

$$
Z=f(T, p) .
$$

According to the experiment above, $n$ groups of $Z$-factor and $(T, p)$ can be got, namely, $n$ groups of training samples,

$$
S=\left\{\left(x_{1}, Z_{1}\right),\left(x_{2}, Z_{2}\right), \ldots,\left(x_{n}, Z_{n}\right)\right\},
$$

where $x_{i}=\left[T_{i}, p_{i}\right]$.

Substituting $\left(X_{i}, Z_{i}\right)$ into type (7) and solving the optimization problem in accordance with the formulas (8)-(9), then the optimal solution of Lagrange multiplier is obtained:

$$
\alpha=\left(\alpha_{1}, \alpha_{2}, \ldots, \alpha_{n}\right)^{T} .
$$

Finally the mapping function is got:

$$
Z=\omega \cdot \varphi(T, p)+b,
$$

where

$$
\begin{aligned}
& \omega=\sum_{i=1}^{n} \alpha_{i} \cdot \varphi\left(T_{i}, p_{i}\right) \\
& b=Z_{j}-\sum_{i=1}^{n} \alpha_{i} \cdot K\left(\left(T_{i}, p_{i}\right) \cdot\left(T_{j}, p_{j}\right)\right)+e_{j} .
\end{aligned}
$$

4.3. Prediction Results and Error Analysis of the MM ZFactor. This paper takes $\mathrm{MM}$ as an example, and $Z$-factor prediction model of MM is established. The experimental data of Tables 1-5 were used as the training samples of LSSVM prediction model. Through the model optimization, the $Z$ factor prediction function of MM can be got finally:

$$
Z=Z(T, p)=\sum_{i=1}^{137} \alpha_{i} K\left((T, p),\left(T_{i}, p_{i}\right)\right)+0.1014
$$

Lagrange multiplier of the prediction function is shown in Table 6.

In order to verify the accuracy of $Z$-LSSVM function, $Z$ factor on the $T-p$ plane $(T=600 \mathrm{~K} \sim 800 \mathrm{~K}, p=1.3 \mathrm{MPa} \sim$ $2.25 \mathrm{MPa}$ ) was calculated by this function and compared with the published data. Prediction and comparison results are shown in Tables 7-11.

Figures 2-6 are $Z$ - $p$ diagram of MM and relative error in $600 \mathrm{~K}, 650 \mathrm{~K}, 700 \mathrm{~K}, 750 \mathrm{~K}$, and $800 \mathrm{~K}$.

It can be seen from Tables 7-11 and Figures 2-6 that the predicted results are ideal, and the average relative error is about $2.14 \%$. The maximum relative error in supercritical region is within $5 \%$. The precision of $Z$-LSSVM model was verified. 
TABLE 6: Lagrange multiplier of Z-LSSVM function for MM.

\begin{tabular}{lccccccccc}
\hline$\alpha_{1 \sim 14}$ & $\alpha_{15 \sim 28}$ & $\alpha_{29 \sim 42}$ & $\alpha_{43 \sim 56}$ & $\alpha_{57 \sim 70}$ & $\alpha_{71 \sim 84}$ & $\alpha_{85 \sim 98}$ & $\alpha_{99 \sim 112}$ & $\alpha_{113 \sim 126}$ & $\alpha_{127 \sim 137}$ \\
\hline-1.448 & 2.811 & -2.156 & 2.5334 & 1.737 & -4.433 & -1.941 & 1.174 & -0.686 & -1.076 \\
-1.613 & 2.849 & -1.159 & 2.8383 & 0.531 & -4.898 & -2.294 & 2.14 & 1.936 \\
-1.684 & 1.85 & -0.973 & 2.7659 & 1.454 & -5.981 & -2.203 & 1.5768 & 1.242 \\
0.129 & 1.551 & -0.896 & 0.9347 & 1.596 & -7.121 & -2.076 & 2.0300 & 0.357 & 0.2555 \\
-0.077 & 1.761 & -1.561 & 1.8095 & 1.2061 & -8.991 & -2.083 & 2.7917 & -0.133 & -2.151 \\
0.58 & -0.024 & -0.642 & 0.7623 & 1.9102 & -9.428 & -1.067 & 1.042 & -0.04 & -2.451 \\
1.209 & 2.941 & 3.229 & 1.6540 & 0.7967 & -9.472 & 1.6258 & 5.4064 & -0.136 \\
1.184 & 4.076 & -2.202 & 1.88 & 1.7198 & -10 & -0.547 & 3.9754 & 0.268 & -0.517 \\
0.592 & 5.806 & -3.437 & 3.0374 & 1.2945 & -10.8 & -1.449 & 5.295 & 1.3083 \\
1.686 & 7.071 & -2.118 & 5.1369 & -1.033 & 1.49 & -1.516 & 4.0329 & 0.7572 & 0.0947 \\
1.114 & 10.346 & -0.282 & 2.5213 & -1.385 & 0.7875 & -1.044 & -8.392 & 0.2802 & 0.4235 \\
1.365 & 5.205 & 0.899 & 3.7976 & -2.520 & 1.4125 & -1.106 & -5.882 & 0.6488 \\
2.382 & -0.305 & 1.480 & 3.821 & -3.610 & -0.829 & -1.042 & -4.644 & -0.054 \\
3.405 & -0.783 & 2.442 & 2.0045 & -4.557 & -1.445 & 0.1749 & -3.499 & -0.324 & \\
\hline
\end{tabular}

$\alpha_{i}$ : Lagrange multiplier.

TABLE 7: Z-factor calculated by Z-LSSVM $(600 \mathrm{~K})$.

\begin{tabular}{lccc}
\hline$p / \mathrm{MPa}$ & $Z_{i}$ & $Z_{i s}$ & $\delta / \%$ \\
\hline 1.3 & 0.879 & 0.879 & 0.02 \\
1.35 & 0.866 & 0.874 & 0.89 \\
1.4 & 0.855 & 0.869 & 1.64 \\
1.45 & 0.845 & 0.864 & 2.19 \\
1.5 & 0.837 & 0.858 & 2.54 \\
1.55 & 0.830 & 0.853 & 2.66 \\
1.6 & 0.826 & 0.848 & 2.53 \\
1.65 & 0.824 & 0.842 & 2.15 \\
1.7 & 0.824 & 0.837 & 1.54 \\
1.75 & 0.826 & 0.831 & 0.68 \\
1.8 & 0.829 & 0.826 & 0.38 \\
1.85 & 0.834 & 0.820 & 1.69 \\
1.9 & 0.841 & 0.815 & 3.18 \\
1.95 & 0.848 & 0.809 & 4.86 \\
\hline
\end{tabular}

TABLE 8: $Z$-factor calculated by $Z$-LSSVM $(650 \mathrm{~K})$.

\begin{tabular}{lccc}
\hline$p / \mathrm{MPa}$ & $Z_{i}$ & $Z_{i s}$ & $\delta / \%$ \\
\hline 1.3 & 0.930 & 0.921 & 0.10 \\
1.35 & 0.919 & 0.918 & 0.13 \\
1.4 & 0.908 & 0.915 & 0.70 \\
1.45 & 0.898 & 0.911 & 1.47 \\
1.5 & 0.889 & 0.908 & 2.15 \\
1.55 & 0.880 & 0.905 & 2.73 \\
1.6 & 0.873 & 0.902 & 3.21 \\
1.65 & 0.867 & 0.898 & 3.54 \\
1.7 & 0.862 & 0.895 & 3.72 \\
1.75 & 0.859 & 0.892 & 3.73 \\
1.8 & 0.857 & 0.889 & 3.53 \\
1.85 & 0.858 & 0.885 & 3.13 \\
1.9 & 0.86 & 0.882 & 2.51 \\
1.95 & 0.864 & 0.879 & 1.65 \\
2 & 0.870 & 0.875 & 0.60 \\
2.05 & 0.878 & 0.872 & 0.62 \\
2.1 & 0.886 & 0.868827 & 1.976576 \\
2.15 & 0.895 & 0.865486 & 3.398556 \\
\hline
\end{tabular}

TABLE 9: Z-factor calculated by Z-LSSVM (700 K).

\begin{tabular}{lccc}
\hline$P / \mathrm{MPa}$ & $Z_{i}$ & $Z_{i s}$ & $\delta / \%$ \\
\hline 1.3 & 0.956 & 0.947 & 0.86 \\
1.35 & 0.950 & 0.945 & 0.52 \\
1.4 & 0.945 & 0.943 & 0.12 \\
1.45 & 0.938 & 0.941 & 0.34 \\
1.5 & 0.931 & 0.939 & 0.85 \\
1.55 & 0.924 & 0.937 & 1.41 \\
1.6 & 0.916 & 0.935 & 2.02 \\
1.65 & 0.909 & 0.933 & 2.65 \\
1.7 & 0.901 & 0.931 & 3.25 \\
1.75 & 0.894 & 0.929 & 3.79 \\
1.8 & 0.888 & 0.927 & 4.23 \\
1.85 & 0.884 & 0.925 & 4.51 \\
1.9 & 0.881 & 0.923 & 4.60 \\
1.95 & 0.880 & 0.921 & 4.46 \\
2 & 0.882 & 0.919 & 4.09 \\
2.05 & 0.885 & 0.917 & 3.51 \\
2.1 & 0.890 & 0.915 & 2.75 \\
2.15 & 0.896 & 0.913 & 1.84 \\
2.2 & 0.903 & 0.911 & 0.86 \\
2.25 & 0.91 & 0.909 & 0.12 \\
\hline
\end{tabular}

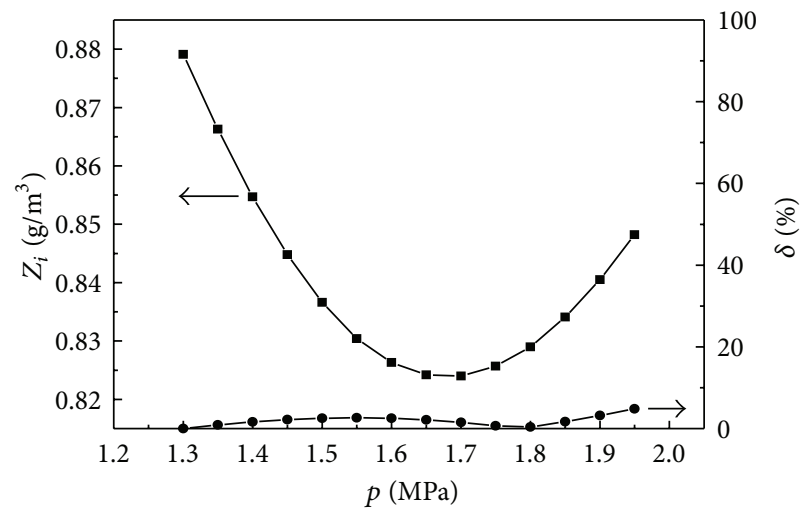

Figure 2: The $Z$ - $p$ diagram and the relative error of MM (600 K).

\section{Conclusion}

The $Z$-factor is an important parameter in the calculation of thermodynamic engineering. Accurate measurement and 
TABLE 10: Z-factor calculated by Z-LSSVM (750 K).

\begin{tabular}{lccc}
\hline$p / \mathrm{MPa}$ & $Z_{i}$ & $Z_{i s}$ & $\delta / \%$ \\
\hline 1.3 & 0.969 & 0.965 & 0.42 \\
1.35 & 0.971 & 0.964 & 0.74 \\
1.4 & 0.972 & 0.963 & 0.95 \\
1.45 & 0.971 & 0.961 & 1.03 \\
1.5 & 0.969 & 0.960 & 0.96 \\
1.55 & 0.966 & 0.959 & 0.69 \\
1.6 & 0.960 & 0.958 & 0.26 \\
1.65 & 0.953 & 0.956 & 0.33 \\
1.7 & 0.945 & 0.955 & 1.05 \\
1.75 & 0.936 & 0.954 & 1.84 \\
1.8 & 0.928 & 0.953 & 2.6 \\
1.85 & 0.919 & 0.951 & 3.40 \\
1.9 & 0.912 & 0.950 & 4.04 \\
1.95 & 0.906 & 0.949 & 4.53 \\
2 & 0.902 & 0.948 & 4.81 \\
2.05 & 0.900 & 0.946 & 4.90 \\
2.1 & 0.9 & 0.945 & 4.78 \\
2.15 & 0.902 & 0.944 & 4.49 \\
2.2 & 0.904 & 0.943 & 4.07 \\
2.25 & 0.908 & 0.942 & 3.57 \\
\hline
\end{tabular}

TABLE 11: $Z$-factor calculated by $Z$-LSSVM (800 K).

\begin{tabular}{lccc}
\hline$p / \mathrm{MPa}$ & $Z_{i}$ & $Z_{i s}$ & $\delta / \%$ \\
\hline 1.65 & 1.002 & 0.972 & 2.99 \\
1.7 & 0.997 & 0.972 & 2.56 \\
1.75 & 0.990 & 0.971 & 1.93 \\
1.8 & 0.982 & 0.970 & 1.18 \\
1.85 & 0.973 & 0.970 & 0.32 \\
1.9 & 0.964 & 0.969 & 0.54 \\
1.95 & 0.955 & 0.968 & 1.36 \\
2 & 0.95 & 0.967 & 2.11 \\
2.05 & 0.940 & 0.967 & 2.74 \\
2.1 & 0.935 & 0.966 & 3.22 \\
2.15 & 0.931 & 0.965 & 3.58 \\
2.2 & 0.928 & 0.965 & 3.81 \\
2.25 & 0.926 & 0.964 & 3.93 \\
\hline
\end{tabular}

calculation of compressibility factor is significant. The measurement at high temperature and high pressure is the focus of the study. During the study, the following conclusions can be obtained:

(1) Based on the actual gas equation of state, the experimental method to measure $Z$-factor at high temperature and pressure state is presented. $Z$-factor value in superheated and supercritical region can be accurately measured by this method, and the method is relatively simple and easy to operate.

(2) Z-factor prediction method based on LSSVM has been proposed. With this method, the $Z$-factor prediction model of MM has been established, and the empirical formula of $Z$-factor is obtained. The experiments show that the average relative error of

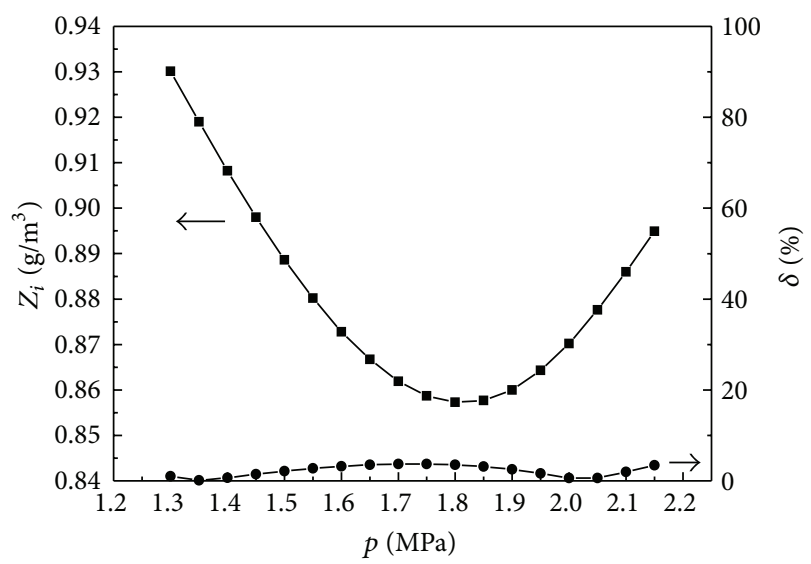

Figure 3: The $Z$ - $p$ diagram and the relative error of MM ( $650 \mathrm{~K})$.

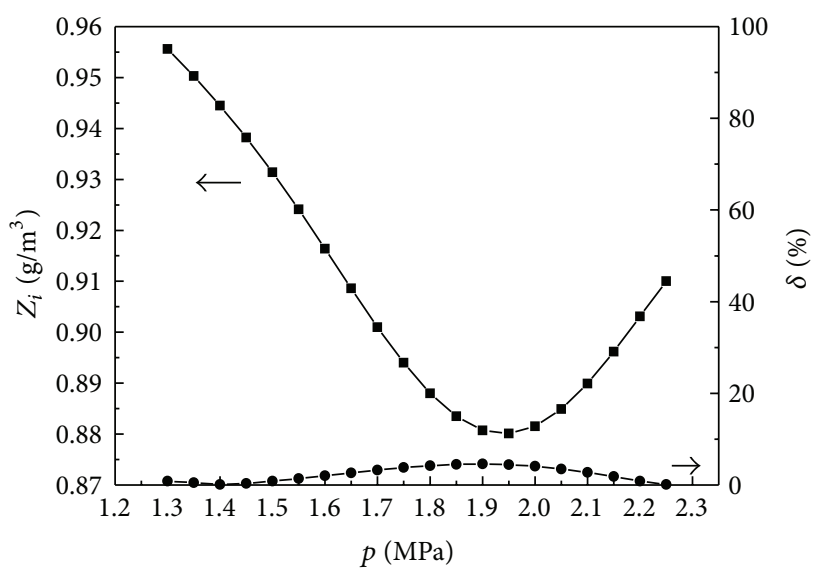

Figure 4: The $Z$ - $p$ diagram and the relative error of MM (700 K).

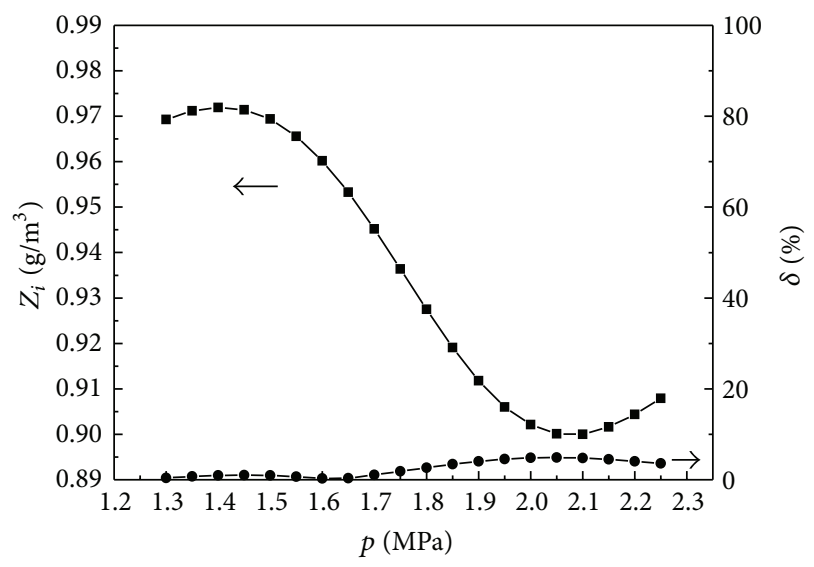

Figure 5: The $Z$ - $p$ diagram and the relative error of MM (750 K).

the prediction model is $2.14 \%$ and the precision of $Z$ LSSVM method has been verified.

(3) The method proposed in this paper is universal and can be widely used for other organic working fluids. Besides, it should also have the same accuracy to measure and predict the density of organic working fluids. 


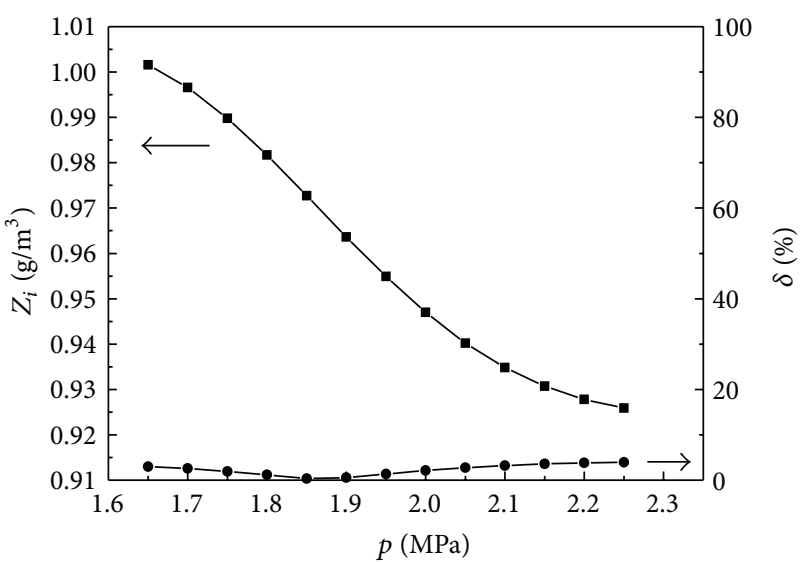

Figure 6: The $Z$ - $p$ diagram and the relative error of MM (800 K).

\section{Competing Interests}

The authors declare that they have no competing interests.

\section{Acknowledgments}

This work was sponsored by the Fundamental Research Funds for the Central Universities (2015MS102).

\section{References}

[1] N. Azizi, R. Behbahani, and M. A. Isazadeh, "An efficient correlation for calculating compressibility factor of natural gases," Journal of Natural Gas Chemistry, vol. 19, no. 6, pp. 642645, 2010.

[2] A. Kamari, A. Hemmati-Sarapardeh, S.-M. Mirabbasi, M. Nikookar, and A. H. Mohammadi, "Prediction of sour gas compressibility factor using an intelligent approach," Fuel Processing Technology, vol. 116, pp. 209-216, 2013.

[3] A. Chamkalani, S. Zendehboudi, R. Chamkalani, A. Lohi, A. Elkamel, and I. Chatzis, "Utilization of support vector machine to calculate gas compressibility factor," Fluid Phase Equilibria, vol. 358, pp. 189-202, 2013.

[4] H. W. Xiang, The Corresponding States Principle and Its Practice, Elsevier Science \& Technology, 2005.

[5] M. A. Mahmoud, "Development of a new correlation of gas compressibility factor (Z-factor) for high pressure gas reservoirs," in Proceedings of the North Africa Technical Conference \& Exhibition, Cairo, Egypt, April 2013.

[6] P. M. Dranchuk and J. H. Abou-Kassem, "Calculation of Z factors for natural gases using equations of state," Journal of Canadian Petroleum Technology, vol. 14, no. 3, pp. 34-36, 1975.

[7] J. R. Travis, D. Piccioni Koch, J. Xiao, and Z. Xu, "Real-gas equations-of-state for the GASFLOW CFD code," International Journal of Hydrogen Energy, vol. 38, no. 19, pp. 8132-8140, 2013.

[8] F. R. Hilgeman, G. Bertrand, and B. Wilson, "Using Dalton's law of partial pressures to determine the vapor pressure of a volatile liquid," Journal of Chemical Education, vol. 84, no. 3, pp. 469470, 2007.

[9] C. Jianming and L. Genbao, Advanced Engineering Thermodynamics, Peking University Press, 2010.
[10] R. Abbas, A. Schedemann, C. Ihmels, S. Enders, and J. Gmehling, "Measurement of thermophysical pure component properties for a few siloxanes used as working fluids for organic rankine cycles," Industrial and Engineering Chemistry Research, vol. 50, no. 16, pp. 9748-9757, 2011.

[11] R. Abbas, E. C. Ihmels, S. Enders, and J. Gmehling, "Measurement of transport properties for selected siloxanes and their mixtures used as working fluids for organic rankine cycles," Industrial \& Engineering Chemistry Research, vol. 50, no. 14, pp. 8756-8763, 2011.

[12] V. Vapnik, "An overview of statistical learning theory," IEEE Transation on Neural Network, vol. 10, no. 5, pp. 988-999, 1999.

[13] F. Ruiming, Theory and Application Analysis of Support Vector Machine, Electric Power Press, Beijing, China, 2007 (Chinese).

[14] J. A. K. Suykens and J. Vandewalle, "Least squares support vector machine classifiers," Neural Processing Letters, vol. 9, no. 3, pp. 293-300, 1999.

[15] X. Wang, J. Chen, C. Liu, and F. Pan, "Hybrid modeling of penicillin fermentation process based on least square support vector machine," Chemical Engineering Research and Design, vol. 88, no. 4, pp. 415-420, 2010.

[16] Z.-H. Han and X.-X. Zhu, "Selection of training sample length in support vector regression based on information entropy," Proceedings of the Chinese Society of Electrical Engineering, vol. 30, no. 20, pp. 112-116, 2010. 


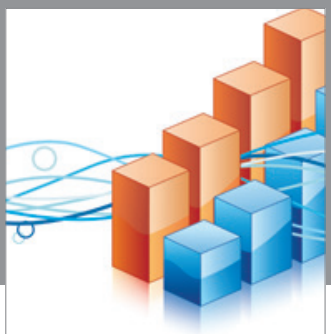

Advances in

Operations Research

vatem alat4

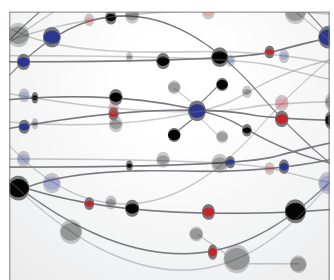

\section{The Scientific} World Journal
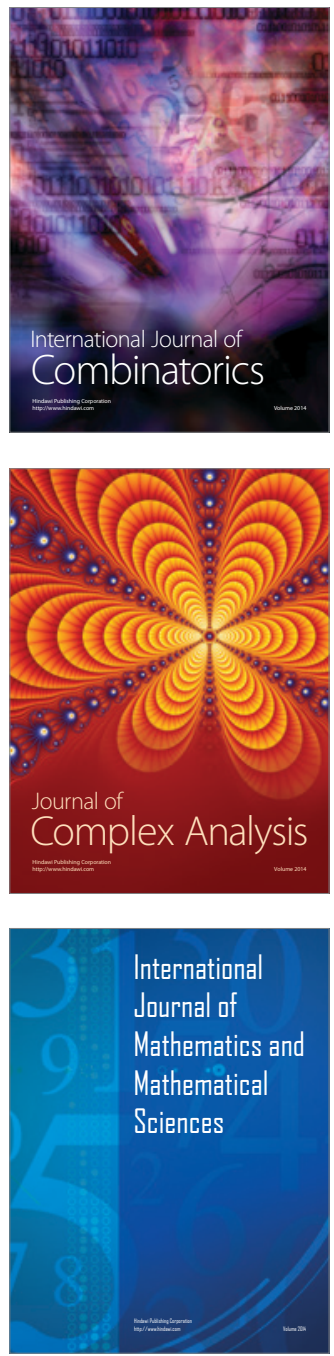
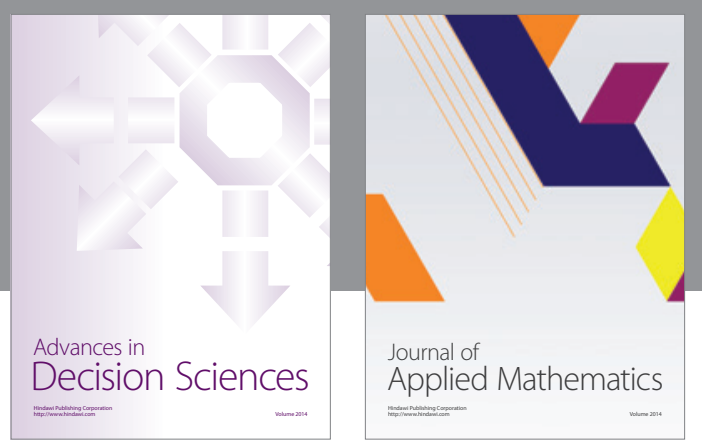

Algebra

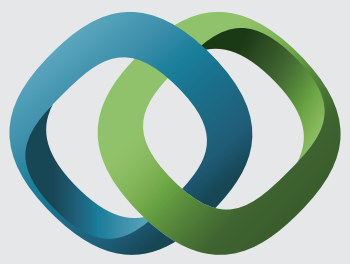

\section{Hindawi}

Submit your manuscripts at

http://www.hindawi.com
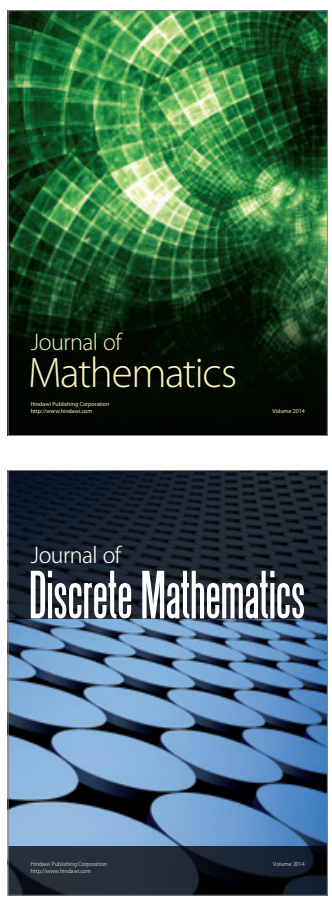

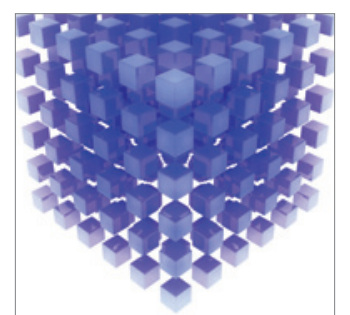

Mathematical Problems in Engineering
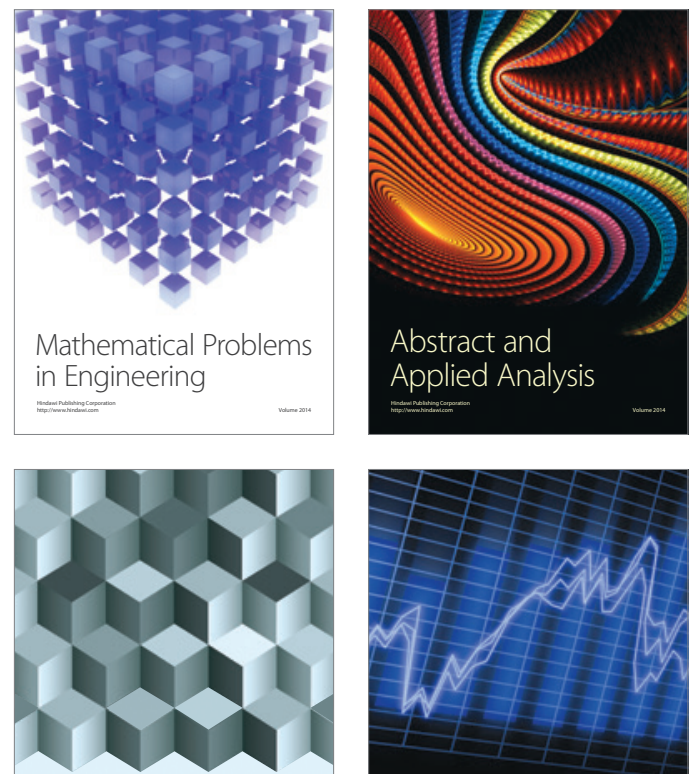

Journal of

Function Spaces

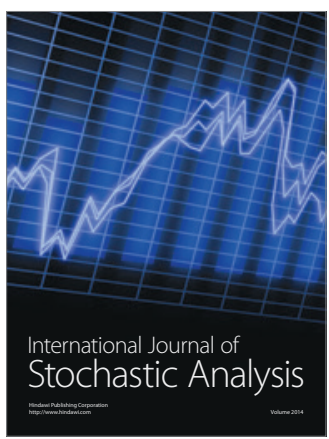

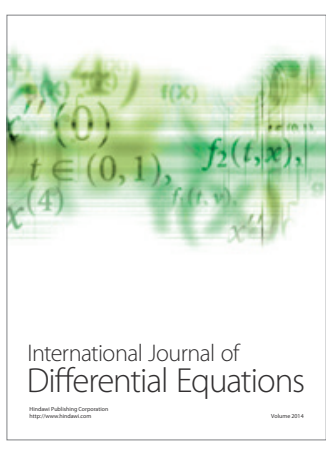
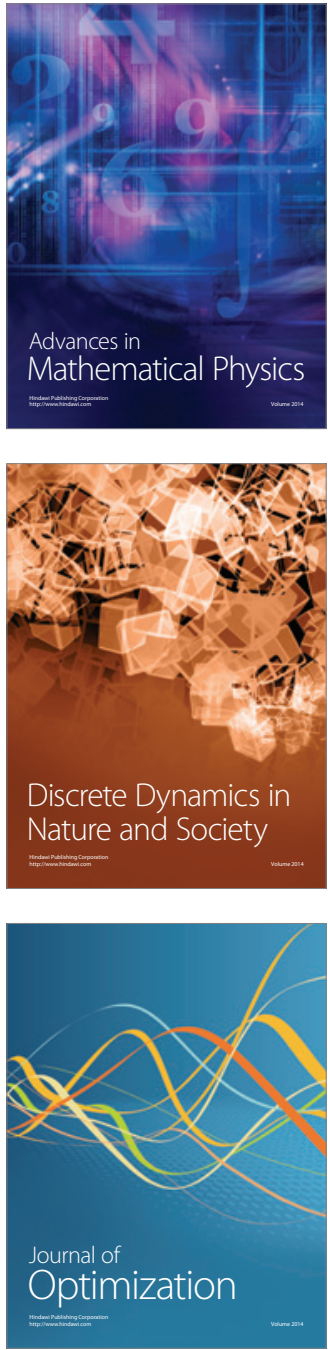